\title{
(C) OPEN ACCESS \\ Redesigning care: adapting new improvement methods to achieve person-centred care
}

\author{
Onil Bhattacharyya, ${ }^{1,2}$ David Blumenthal, ${ }^{3}$ Roger Stoddard, ${ }^{4}$ \\ Lynne Mansell, ${ }^{5}$ Kathryn Mossman, ${ }^{6}$ Eric C Schneider ${ }^{3}$
}

For numbered affiliations see end of article.

\section{Correspondence to}

Dr Onil Bhattacharyya, Family and Community Medicine Women's College Hospital, Toronto, ON M5S 1B2, Canada; onil.bhattacharyya@wchospital.

Received 13 April 2018 Revised 14 August 2018 Accepted 21 August 2018 Published Online First 21 September 2018
Check for updates

(C) Author(s) (or their employer(s)) 2019. Re-use permitted under CC BY-NC. No commercial re-use. See rights and permissions. Published by BMJ.

To cite: Bhattacharyya $O$, Blumenthal D, Stoddard R, et al. BMJ Qual Saf 2019:28:242-248.
In many industries, meeting the needs of customers can mean the difference between thriving and going out of business. In the last century, manufacturers started to use and refine methods to reliably make products that offered a better customer experience at lower cost, by reducing defects and waste. ${ }^{1}$ These methods, introduced to healthcare almost 30 years ago ${ }^{2}$, are now part of the routine operations of many hospitals and large physician organisations. They have contributed to improvements in patient experience, reductions in hospital acquired infections and fewer readmissions. ${ }^{3}$ Customer-focused companies have not stood still during this time, creating an abundance of new products and services. ${ }^{45}$ Many of these were made by technology startups and firms adapting industrial design methods like 'human-centred design' to create better experiences by understanding and responding to unspoken or unmet needs of customers. ${ }^{6}$ Embracing methods to create new products and services can augment current efforts to improve existing products and services and has potential to make health systems more responsive to patient's needs. While the financial pressure to improve the user experience is less in healthcare than other service industries, the rise of value-based financing in many health systems creates an incentive to explore new models of care that offer a better patient experience and better health outcomes at lower cost.

The broad goal of improving value and delivering a better experience can be pursued using established methods like Lean and Six-Sigma that are relatively well established in healthcare. ${ }^{7}$ However, these methods may not be as well suited to generating novel services. Methods less familiar to healthcare like design thinking (first introduced by some healthcare organisations in the last 15 years or so), ${ }^{89}$ and Lean Startup (introduced within the last 5 years $)^{10}$ may confer advantages on organisations prepared to pursue them. The use and distinct contributions of these methods may not be clear to most healthcare professionals in leadership roles or on the front lines. This paper explains how more traditional and newer improvement methods can contribute to healthcare delivery. It describes distinct strengths and common features of the different methods and illustrates how they can be combined to achieve higher performance. Last, it notes how leading healthcare organisations have introduced and taken advantage of these novel methods to improve care in general and the patient experience in particular.

\section{RESPONDING TO THE CHALLENGE OF IMPROVING PERSON-CENTRED CARE}

Organisations striving to deliver person-centred care can follow different paths depending on the degree of change they feel is required. They can focus on their core services by modifying the current care delivery approaches to make them more person-centred while retaining most of the existing processes, systems and stakeholders (figure 1). Alternatively, they can create new services that are either adjacent to current activities but work within the existing delivery model, or are transformational and represent a new delivery model, and require new processes, systems and stakeholders. For example, Geisinger Health System in the USA created a programme for patients with poorly controlled diabetes, food insecurity and poverty that provides free food for 10 meals a week for their families 


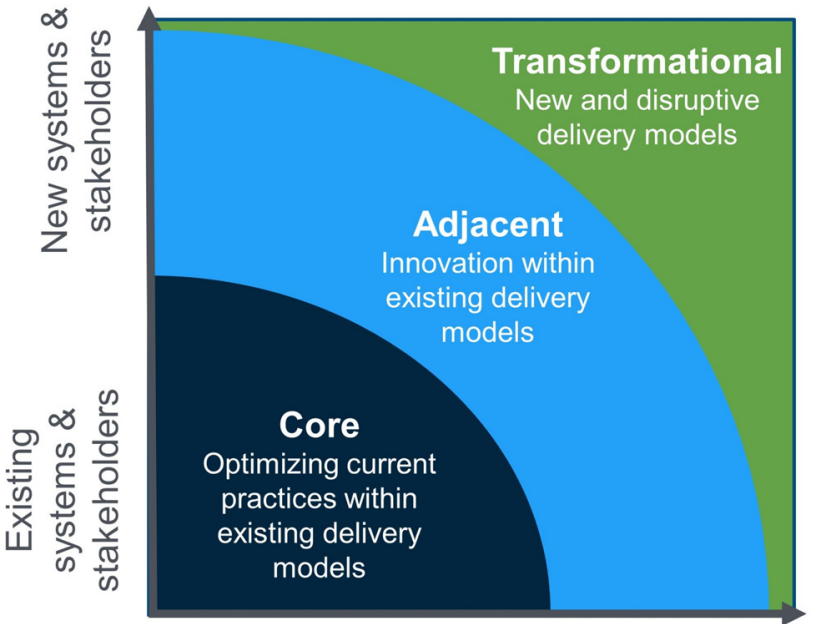

Existing process \& service improvement

New processes \& services

Figure 1 Improving existing services versus creating new services. Adapted from Nagji B, Tuff G. Managing your innovation portfolio. Harvard Business Review. May 2012.

along with group classes on diabetes self-management. This required new processes and new partners like supermarkets, and though it costs $\$ 2200$ per year per patient to administer, it has reduced their total cost of care to the Geisinger Health Plan by more than twice this amount and while substantially reducing A1C. ${ }^{11}$ Creating new services has a distinct set of challenges from improving existing services. Service development methods can be grouped into three approaches, each with a different set of starting assumptions (table 1). The process-focused approach is best suited when an existing service meets a defined customer need but that the production process for delivering that service could be improved (column A). The solution focused approach is helpful when a new service concept may better meet the needs of customers than existing options and this may require entirely new processes (column B). The problem or need-focused approach is best when customers have unmet needs that are not well defined but could be met by new, and not yet developed, services that may or may not relate to current activities (column C).

These methods have some overlap, but also different strengths in making care more person-centred, and they depend on how open an organisation is to moving beyond its core activities. All three methods (process-focused, solution-focused and problem or need-focused) are iterative, but they vary in the degree of openness to changing key features, the target user or the goals of the service across iterations. They all involve some elements of idea generation, prototyping and user testing but they have a different emphasis. Problem or need-focused approaches are particularly good at generating novel options that are not obvious at the outset. Solution-focused approaches are particularly good at rapidly testing core elements of a new service concept in context and modifying them to determine if they provide some notional value and have potential for growth. Process-focused approaches are good for understanding flaws in current services and reliably testing the impact of minor modifications on predefined performance measures.

Table 1 Comparing methods for developing person-centred services

\begin{tabular}{|c|c|c|c|}
\hline Initial focus & A. Process & B. Solution & C. User need or problem \\
\hline Key a & $\begin{array}{l}\text { Existing service meets a defined customer } \\
\text { need but production process can be } \\
\text { improved }\end{array}$ & $\begin{array}{l}\text { A new service could better meet the needs } \\
\text { of existing or new customers, which may } \\
\text { be independent of current processes }\end{array}$ & $\begin{array}{l}\text { Customers have unmet needs which are } \\
\text { not well defined but could be met by new } \\
\text { services with new processes that have yet } \\
\text { to be developed }\end{array}$ \\
\hline $\begin{array}{l}\text { Circumstances } \\
\text { where method is } \\
\text { most appropriate }\end{array}$ & $\begin{array}{l}\text { Established service that meets users needs } \\
\text { when delivered appropriately. There is } \\
\text { already a service with users that with their } \\
\text { needs } \\
\text { There is a defined production process but } \\
\text { it is not consistently achieving the desired } \\
\text { outcomes }\end{array}$ & $\begin{array}{l}\text { There is an idea for a new service, but no } \\
\text { defined processes } \\
\text { There are no existing customers } \\
\text { The fit between the solution and a user } \\
\text { need or problem has not been confirmed }\end{array}$ & $\begin{array}{l}\text { There are a set of potential customers who } \\
\text { are poorly served but whose needs are not } \\
\text { well understood } \\
\text { There is no idea for a new service and } \\
\text { there are no defined processes to build on }\end{array}$ \\
\hline Desired endpoint & Refined production process & $\begin{array}{l}\text { Working version of new service, proposed } \\
\text { processes }\end{array}$ & $\begin{array}{l}\text { Customer need defined, new service } \\
\text { defined, service prototype generated }\end{array}$ \\
\hline Key steps & $\begin{array}{l}\text { Define sources of unnecessary variation or } \\
\text { waste and analyse root cause } \\
\text { Devise strategy to improve process, plan and } \\
\text { implement } \\
\text { Measure outcome } \\
\text { Standardise approach if effective or modify } \\
\text { if not }\end{array}$ & $\begin{array}{l}\text { Build minimally functional version of } \\
\text { service based on initial concept } \\
\text { Test with potential users } \\
\text { Modify service or target group to find fit } \\
\text { between user need and proposed service } \\
\text { Test ways to expand }\end{array}$ & $\begin{array}{l}\text { Interview and observe users and immerse } \\
\text { in their context to create empathy } \\
\text { Consider analogous situations from other } \\
\text { industries } \\
\text { Characterise problem and generate wide } \\
\text { range of solutions } \\
\text { Narrow options and present mock-ups to } \\
\text { users to identify which are promising }\end{array}$ \\
\hline Early champion & Manufacturing & Technology startups and software & Industrial design \\
\hline
\end{tabular}


Box 1 Process-focus: improving patient flow and responsiveness in primary care

Virginia Mason's Kirkland clinic wanted to improve responsiveness, patient flow and physician workload at their primary care clinic. They convened a Rapid Process Improvement Workshop team that studied the workflow of one physician for 3 days, asking her to try different sequences and physical layouts, timing each variation with the aim of eliminating waste. Each modification was planned, implemented, analysed and further refined where the results showed benefit. They found that instead of saving paperwork for the end of the day, it was more efficient for a physician to work with a medical assistant to address small amounts of pertinent paperwork between patient visits. They refined this process to include a flow station, a counter with filing slots where pieces of paperwork could be filed and organised. This freed up time for other tasks, including phone calls. As a result, patient satisfaction with phone access went from $40 \%$ to $85 \%$, and incoming calls went down by 50000 each year because calls were more often answered the first time.

Adapted from: Plsek P. Accelerating Health Care Transformation with Lean and Innovation: the Virginia Mason experience. CRC Press, Boca Raton, 2013.

\section{A process-focused approach to consistently deliver a service with existing users}

When a person-centred set of services is offered, but delivery is inconsistent or suboptimal, process improvement methods from the manufacturing industry like Lean can be effective (column A in table 1). These methods have been implemented in many organisations over the last 20 years. ${ }^{12}$ They involve identifying sources of variation, planning small tests of change, implementing them, studying the result and acting on the findings. When the core elements of the service are appropriate and target the right group with clear outcomes, process improvement can improve efficiency and patient experience. The focus is on iteratively modifying a small number of parameters, and it is best applied when there is a comparatively high likelihood of success, and improvements can be found with changes in the process, as shown in the example in box 1. Lean methods are particularly good at determining whether a service is improving based on a specific change, and the evaluation methods are designed to pick out small effects that justify the tweak in services by highlighting trends over time or across sites.

\section{A solution-focused approach to test service options for fit with a user need \\ When a new service idea is available but has not yet been tested, a solution-focused approach like Lean Startup may be useful (column B). Lean Startup is a close cousin of the Lean method, pioneered by startup companies who faced the uncertainty of creating a new service whose potential customers were not yet known. The}

key distinction between Lean and Lean startup is that Lean methods focus on making an existing service with known customers more reliable and higher quality, while Lean Startup focuses on creating a new service that some group will want. It is similar to Joseph Juran's concept of quality planning - 'define customers and how to meet their needs', ${ }^{13}$ but this approach has recently undergone extensive development in the technology industry, particularly by small companies trying to develop a product or service someone is willing to pay for before they run out of money. ${ }^{5}$ Until you have a defined and interested customer base, it is difficult to define quality or determine what should be reliably delivered. To test the fit between a proposed solution and a potential user need, serial entrepreneur Eric Ries suggests: 'We must learn what customers really want, not what they say they want or what we think they should want'. ${ }^{4} \mathrm{He}$ suggests the best way to understand what customers really want is by rapidly building a prototype that has enough functionality for potential customers to use it and give feedback.

This minimum viable product is tested iteratively, modifying its features to suit different groups of customers until there is a good fit between the service and a customer need. For example, Dropbox founder Drew Houston had difficulty describing his concept for seamless file sharing, because file synchronisation was not a problem most people knew they had, and he knew it would be very challenging to develop. ${ }^{14}$ So he made and released a video of someone using a mock-up of Dropbox, many people viewed it and 75000 people signed up for a beta version. This required no upfront investment in technology, but it signalled sufficient interest in the solution for him to raise the funds and put the necessary work in to build it. Ideally, a minimum viable product serves at least one specific audience, addresses at least one key problem, has a well-designed user experience and is easy to build and launch quickly. ${ }^{15}$ The Lean Startup method is most useful when starting with a novel solution and trying to discover whether anyone wants it (see box 2). To do this, it may need to either change the group it targets, target a different need in the same group or modify the solution before it finds a combination that works. The key outputs of Lean Startup are a service and a business model, and it focuses on testing the value and the growth potential of the service.

Problem-focused approach to generate service options If patient needs are poorly understood and there are no ideas for a solution to test, then problem-focused methods like human-centred design or experience-based co-design may be appropriate (column C). With origins in industrial design, these methods seek to elicit user needs and generate service options that may not currently be available. ${ }^{1617}$ They use interviews, observation and immersion in a user's context to create empathy and uncover unspoken and 
Box 2 Solution-focus: Creating a virtual hospital at Women's College Hospital

Women's College Hospital is an ambulatory care facility in Toronto which has declared in its strategic plan that it will provide $25 \%$ of care virtually by 2022 . It has defined virtual care (synchronous or asynchronous interactions between patients and providers using voice, video or text) as a solution, but it was not clear which patients to target, which clinical model to use, what technologies to adopt and what the benefit should be. This solutionfocused approach requires testing on a small scale across a range of services, with a range of patient needs to see where there is a fit. They are beginning with follow-up for same day knee surgery, video visits in gastroenterology and wound care follow-up in dermatology. In each case, they will test demand for each service by advertising it with a small number of patients and providers to see how many sign up and iterate on target group and feature of the service until there is sufficient interest to build and launch a working service with those features. The goal is first to build a service that patients and providers will want, articulate key hypotheses around its value and test them iteratively to determine whether the hypotheses hold and what its benefit will be. This could include improved access to care, patient experience, provider caseload, revisit rates, reduced use of outside services and improved control of chronic conditions. But it is unclear at the outset which of these will be demonstrated in early tests and which of these are sufficient to justify continuing or expanding the service. They will also track how to grow these services, considering different staffing models and approaches to remuneration.

unrecognised needs of customers. ${ }^{18}$ They also explore analogous situations in other contexts to identify potential solutions. For example, airlines have increasingly shifted tasks to customers in ways that have increased efficiency of the check-in process, without hampering the user experience. This insight could be applied to healthcare settings through self check-in, inputting or confirming demographic information in the chart and filling out standardised screening tools and symptom scores before their appointment. Though it also involves rapid prototyping like Lean Startup, the relative strength of human-centred design is in defining individual motivations and the problems to be solved in order to generate a range of promising service options that can then be tested. This is highlighted in the example in box 3 .

The methods described above are usually applied independently, but could be used sequentially to create and scale novel solutions for previously unrecognised problems. For example, Penn Medicine in the USA observed that their employees often used the emergency department for minor health problems and approached their Center for Health Care Innovation
Box 3 Problem or need focus: community health transformation at Mayo Clinic

The Mayo Clinic Centre for Innovation was assigned to reassess their community primary care model to prepare for value-based funding. The project was led by two industrial designers who did not start with a well-framed problem, but rather brought an open mind to explore the current state of primary care and its potential. They started with scanning and framing and spent time observing and questioning patients and providers in Kasson Clinic in Dodge County, a rural community in Minnesota. They noted that providers did not function as a team and that many patients presented with issues which required social services, rather than heath care. They explored the range of patient needs and what providers could address them, and they brought in a community engagement coordinator to establish a partnership with Dodge County. They held forums and workshops in local cafes and an assisted living community. They handed patients in the waiting room sheets with the title: 'I wish Kasson Clinic had...' to better understand what might be helpful. In the second phase of the project, they began to test assumptions about what were important problems in order to generate potential solutions. They handed sheets to physicians that asked 'Who should have been in that appointment today?' and noted that for every five patients seen by the doctor, only one needed to be seen by a physician, three could be managed by another team member and one did not require visit-based care. This led to a series of experiments around team composition with different combinations of providers (doctor, nurse practitioner or physician assistant), registered nurses, licensed practice nurses, medical assistants and a scheduler. Once they had found a team model that had positive feedback and promise, they were ready to deploy a working prototype in the clinic. Initial testing demonstrated the ability to shift activity to non-physician team members and for the community engagement coordinator to address non-medical concerns through community partners.

Adapted from: Ryan A, 2016. Transforming Community Health Through Systemic Change. https://medium.com/the-overlap/transformingcommunity-health-through-systemic-design-5b22b9d5bf

for assistance (see box 4). The first phase helped define the problem (people have a range of health needs to address throughout the day, and getting to a clinic is inconvenient) and generate a potential solution (the FirstCall application). In the second phase, they quickly launched a minimum viable product to identify the core features and determined that it met a patient need and they tested ways it could scale. The next phase is to improve the process they have just developed to standardise communication between nurses and doctors, to reduce variation in clinical responses 
Box 4 Sequential methods to develop and spread a new service to improve access to care for hospital employees at Penn Medicine

Penn Medicine, a large self-insured US health system, observed that employees often used the emergency department (ED) for minor problems rather than contacting primary care physicians, who had long waits for appointments. This health system had established quality improvement teams as well as the Penn Medicine Center for Health Care Innovation, which is staffed with project managers, user experience designers and software developers, so it was skilled in a wide range of methods.

\section{Defining the problem}

Employees currently access services in-person, so they established a temporary walk-in clinic with existing resources to observe patient behaviour and needs in the context of seeking clinical care. Thirty-two patients came in the first month, with a range of acute clinical conditions, many of which did not require in-person care. The team used contextual inquiry and employee interviews to determine drivers of avoidable ED utilisation. They found that the ED was often used instead of primary care because employees wanted same-day care and advice, one-stop shopping that included labs and prescriptions, and 24/7 convenience. They also found that people would rather not have to go to a clinic in person if it could be avoided.

\section{Developing and testing a minimum viable product}

Based on these learnings, the innovation centre team quickly designed a service that allowed employees to reach nurses and doctors by phone from any location. The service could triage patient complaints and provided same-day access to labs, remote prescribing and in-person appointments across the region as needed. The team prototyped the new service and tested it with a few employees to see if there was a fit. The initial response was promising, so they created a web-based application connecting patients to nurses $24 / 7$, to which they added physician backup and launched on a small scale. They advertised phone, chat and video visits, but only phone communication was built out in entirety. By tracking the number of requests for other communication modalities, the team found little demand for chat and video, so they never built them. The service received a total of 217 calls over a 3-month period, and $24 \%$ of respondents said that they would have gone to the ED if they did not have this service.

\section{Testing a growth model for the new service} Initial call volumes were low, but after advertising was pushed out to a greater number of employees, calls more than doubled within a month. The team recognised that they needed to match supply with demand as they grew. They tried nurses with physician back-up, nurse

Continued

\section{Box 4 Continued}

practitioners who needed less back-up, a fixed physician on call or an Uber-like physician pool that nurses could draw on as needed. These experiments were run in parallel, looking for a signal of increased ability to handle the rising demand in each arm.

\section{Standardising and integrating the new process into} the core operations of the organisation Now that the service was running, appeared to meet a need and could scale, the team applied process improvement methods to standardise communication within the service, given the variation in clinical responses to similar problems and response times. They also started on the greater operational challenge-moving this from a pilot programme to something owned by the institution and part of its core functioning.

to similar problems and response times and turn the initiative into a core function of the institution.

\section{HOW CAN HEALTHCARE ORGANISATIONS TAKE ADVANTAGE OF THE FULL RANGE OF METHODS?}

While problem, solution and process-oriented methods have different starting points, the example from Penn above illustrates that in some cases they can be applied in sequence to generate more person-centred services that are scalable. Most healthcare organisations are using process improvement, and some have started to apply problem-oriented approaches like user-centred or experience-based codesign, but relatively few use solution-oriented ones like Lean Startup. This is in part because the method is new (the seminal text was published in 2011) ${ }^{4}$, and because the application of these entrepreneurial functions within large organisations is even more recent (case studies from the US government, GE, and others were published in 2017). ${ }^{19}$

Building these capabilities will require either partnering with other organisations, hiring new types of staff, or training existing staff in these methods, as has been done for quality improvement. Partnership can bring groups with expertise in Lean Startup into the organisation, such as accelerators who support startup companies that are developing new products and services. In the USA, the Cedars-Sinai delivery system and the Techstars accelerator have brought in and mentored startups who work in priority areas for the health system, often leading the development of new services or procurement of new technologies in the hospital, and there are emerging examples of this type of partnership in Canada ${ }^{20}$ and the UK. Organisations can hire new staff such as user experience designers, app developers, engineers or entrepreneurs in residence, who are often situated in 'innovation centres'. This model has been used in the USA, first 
at Mayo Clinic, and also in the UK (Helix Centre at Imperial College London) and in Canada (Centre for Global eHealth innovation at the University Health Network in Toronto). To train current staff, UPenn has added human-centred design (HCD) methods to their quality improvement training programme, while Virginia Mason has trained all staff in the basics of experienced-based codesign, administrators at an intermediate level and over 80 people have received advanced training, without hiring any full time designers.

\section{Implementation challenges-where do these functions fit into healthcare organisations?}

Creating new service lines is a distinct function with distinct methods, so some organisations have separated it from senior leadership and frontline delivery, so they are not constrained by existing policies and processes, while others have integrated them closely to maximise strategic alignment and facilitate uptake. ${ }^{21}$ The degree of integration will depend on the importance of creating new services for leadership and the openness of the staff to iteratively generating and abandoning novel approaches that are not connected to current workflows. Irrespective of the structure, generating new services is very challenging and skill at need and solution-focused methods can help manage the uncertainty around the effectiveness of what is proposed and how it affects different groups. ${ }^{22}$ Human-centred design can help understand what key stakeholders value and Lean Startup can help test a value proposition for a range of potential customers or stakeholders by launching a product or service on a small scale and iterating until you find a fit with one or more groups. ${ }^{23}$ For example, services like remote monitoring may be valued by patients because of the reduced need to go in to clinic, but providers may worry about the increase in workload, institutions may worry about potential liability and payers may worry about the cost of patient facing digital tools. Lean Startup does not resolve this tension, but allows for iteration in the features of the technology, clinical model and business model that could optimise the value proposition for patients and one or more other groups to facilitate uptake by key stakeholders. In one example of this, the Johns Hopkins Bayview Medical Centre applied design thinking, Lean startup and Lean Six Sigma techniques to their heart failure model of care and was able to improve the experience of care and reduce heart failure readmission rates, saving payers almost $\$ 1$ million annually, much lower than the \$200 000 annual cost of implementation. ${ }^{24}$ The mix of changing public expectations, new financing models and novel technologies create new possibilities for health services and organisations that have the skills to conceptualise, prototype and refine new models of care will be more likely to thrive.
Author affiliations

${ }^{1}$ Frigon Blau Chair in Family Medicine Research, Women's College Hospital Research Institute, Toronto, Ontario, Canada

${ }^{2}$ Family and Community Medicine, Institute of Health Policy Management and Evaluation, University of Toronto, Toronto, Ontario, Canada

${ }^{3}$ The Commonwealth Fund, Policy and Research, New York City, New York, USA

${ }^{4}$ National Leadership Council, Primary and Integrated Health Care Innovation Network, Quispamsis, New Brunswick, Canada

${ }^{5}$ National Leadership Council, Primary and Integrated Health Care Innovation Network, Edmonton, Alberta, Canada

${ }^{6}$ Institute for Health Systems Solutions and Virtual Care, Women's College Hospital, Toronto, Ontario, Canada

Acknowledgements We would like to acknowledge David Asch from the Penn Medicine Center for Health Care Innovation for his detailed description of the FirstCall service.

Contributors $\mathrm{OB}$ wrote the initial draft after discussion with DB and ECS. LM, RS and KM were included in a substantial revision based on editor's comments on a previous version. All coauthors were involved in the subsequent revisions and approved the final draft.

Funding This work was funded by a Harkness Fellowship in Health Policy and Practice from the Commonwealth Fund.

Competing interests None declared.

Patient consent Not required.

Provenance and peer review Not commissioned; internally peer reviewed.

Open access This is an open access article distributed in accordance with the Creative Commons Attribution Non Commercial (CC BY-NC 4.0) license, which permits others to distribute, remix, adapt, build upon this work noncommercially, and license their derivative works on different terms, provided the original work is properly cited, appropriate credit is given, any changes made indicated, and the use is noncommercial. See: http://creativecommons.org/licenses/by-nc/4.0

\section{REFERENCES}

1 Juran JM. Managerial breakthrough. New York, NY: McGrawHill, 1964.

2 Laffel G, Blumenthal D. The case for using industrial quality management science in health care organizations. JAMA 1989;262:2869-73.

3 D'Andreamatteo A, Ianni L, Lega F, et al. Lean in healthcare: a comprehensive review. Health Policy 2015;119:1197-209.

4 Ries E. The lean startup: how today's entrepreneurs use continuous innovation to create radically successful businesses. New York, NY: Currency, 2011.

5 Blank S, 2013. Why the Lean start-up changes everything. Harvard business review. https://hbr.org/2013/05/why-the-leanstart-up-changes-everything (accessed 17 Oct 2016).

6 Brown T. Design thinking. Harv Bus Rev 2008;86:84.

7 D'Andreamatteo A, Ianni L, Lega F, et al. Lean in healthcare: a comprehensive review. Health Policy 2015;119:1197-209.

8 Brennan MD, Duncan AK, Armbruster RR, et al. The application of design principles to innovate clinical care delivery. J Healthc Qual 2009;31:5-9.

9 Roberts JP, Fisher TR, Trowbridge MJ, et al. A design thinking framework for healthcare management and innovation. Healthc 2016;4:11-14.

10 Zuckerman B, Margolis PA, Mate KS. Health services innovation: the time is now. JAMA 2013;309:1113-4.

11 Feinberg A, Slotkin JR, Hess A, 2017. How Geisinger treats diabetes by giving away free, healthy food. Harvard business review. https://hbr.org/2017/10/how-geisinger-treats-diabetesby-giving-away-free-healthy-food (accessed 3 Aug 2018). 
12 Best M, Neuhauser D. Joseph Juran: overcoming resistance to organisational change. Qual Saf Health Care 2006;15:380-2.

13 Ries E, 19 October 2011. How dropbox started as a minimal viable product. Techcrunch. https://techcrunch.com/2011/10/ 19/dropbox-minimal-viable-product/ (accessed 1 Aug 2018).

14 De S, 30 January 2018. How to build an MVP in the right way in 2018. Medium. Available from: https://medium.com/swlh/ how-to-build-an-mvp-in-the-right-way-in-2018-f538df0f2bba [Accessed 31 Jul 2018].

15 Deblois S, Lepanto L. Lean and Six Sigma in acute care: a systematic review of reviews. Int J Health Care Qual Assur 2016;29:192-208.

16 Plattner H, 2010. An introduction to design thinking: process guide. Institute of Design at Stanford. https://dschool-old. stanford.edu/sandbox/groups/designresources/wiki/36873/ attachments/74b3d/ModeGuideBOOTCAMP2010L.pdf (accessed 10 Jul 2018).

17 Design council, 2018. Design methods for developing services. https://www.designcouncil.org.uk/sites/default/files/asset/ document/Design\%20methods\%20for\%20developing\% 20services.pdf (accessed 10 Jul 2018).
18 Robert G, Cornwell J, Locock L, et al. Patients and staff as codesigners of healthcare services. BMJ 2015;350:g7714.

19 Kelson MC. Consumer collaboration, patient-defined outcomes and the preparation of Cochrane Reviews. Health Expect 1999;2:129-35.

20 TEC Edmonton, 2018. Health accelerator. https://www. tecedmonton.com/health-accelerator/ (accessed 9 Jul 2018).

21 Bhattacharyya O, Blumenthal D, Schneider E, 2018. Small improvements versus care redesign: can your organization juggle both? NEJM Catalyst. https://catalyst.nejm.org/jugglecare-redesign-innovation-capacity/ (accessed 27 Jul 2018).

22 Ries E. The startup way: how modern companies use entrepreneurial management to transform culture and drive long-term growth. New York, NY: Currency, 2017.

23 Osterwalder A, Pigneur Y, Bernarda G. Value proposition design: how to create products and services customers want. Hoboken, NJ: Wiley and Sons, 2014.

24 Johnson AE, Winner L, Simmons T, et al. Using innovative methodologies from technology and manufacturing companies to reduce heart failure readmissions. Am J Med Qual 2016;31:272-8. 